\title{
Hydrostatic Equilibrium of a Perfect Fluid Sphere with Exterior Higher Dimensional Schwarzschild Spacetime
}

\author{
J. PONCE DE LEON円 \\ Laboratory of Theoretical Physics, Department of Physics, University of \\ Puerto Rico, P.O. Box 23343, San Juan, PR 00931, USA. \\ NORMAN CRUZZ \\ Departamento de Física, Facultad de Ciencia, Universidad de Santiago de \\ Chile, Casilla 30\%, Santiago, Chile.
}

\begin{abstract}
:
We discuss the question of how the number of dimensions of space and time can influence the equilibrium configurations of stars. We find that dimensionality does increase the effect of mass but not the contribution of the pressure, which is the same in any dimension. In the presence of a (positive) cosmological constant the condition of hydrostatic equilibrium imposes a lower limit on mass and matter density. We show how this limit depends on the number of dimensions and suggest that $\Lambda>0$ is more effective in $4 D$ than in higher dimensions. We obtain a general limit for the degree of compactification (gravitational potential on the boundary) of perfect fluid stars in $D$-dimensions. We argue that the effects of gravity are stronger in $4 D$ than in any other number of dimensions. The generality of the results is also discussed.
\end{abstract}

KEY WORDS: Star model in higher dimension

\footnotetext{
${ }^{1}$ E-mail: jponce@upracd.upr.clu.edu or jpdel1@hotmail.com

${ }^{2}$ E-mail: ncruz@lauca.usach.cl
} 


\section{INTRODUCTION}

Lately the study of higher dimensional space-times has led to important generalizations and wider understanding of general relativity solutions. Of special interest are the black-hole solutions found by Myers and Perry [1] . They generalized the Schwarzschild, Reissner-Nordström and Kerr solutions and discussed the associated singularities, horizons and topologies. The cosmological constant was included in these solutions by Xu Dianyan [2]. Also, Bañados et al [3], in the context of Lovelock's theory, found black-hole solutions where the parity of the dimensions plays an important role. Interior solutions for higher dimensional perfect-fluid have been discussed by Krori et al [4] and Shen et al [5]. Other interior solutions, as well as their physical properties, have been discussed in the context of "matter-from-geometry" theory, in which four-dimensional matter is interpreted as a manifestation of five-dimensional geometry [6, 7].

In this paper we are not interested in obtaining new solutions. Our object here is to study the general conditions for the hydrostatic equilibrium of spherical stars in $D$ dimensions. We focus our attention on effects of extra dimensions. Conversely, we are interested in what limitations observations might put on the number of dimensions. We will show that the increase in the number of dimensions increases the effect of mass. We will see that the degree of compactification'], that is the surface gravitational potential of a perfect fluid star, is maximum for $D=4$.

The plan of this article is as follows. In Sec. 2 we will discuss the effects on gravitational mass and pressure in equilibrium configurations. In Sec. 3 we study the degree of compactification of stars in $D$ dimensions, and present an explicit example that illustrates our findings. In Sec. 4 we summarize our results.

\section{HYDROSTATIC EQUILIBRIUM IN $D$ DIM}

\footnotetext{
${ }^{3}$ The degree of compactification $G M / R^{D-3}$, is a measure of how much mass can be packed in a given volume, without provoking gravitational collapse.
} 


\subsection{The Tolman-Oppenheimer-Volkov equation}

In this section we will obtain the $D$-dimensional version of the TolmanOppenheimer-Volkov equation (TOV) and discuss its consequences on the hydrostatic equilibrium of spherical stars in $D$ dim. With this aim, and following the conventional wisdom, we assume that the form of the physical laws is independent of the number of dimensions. Consequently, we start with the Einstein field equations, which in $D$ dimensions are

$$
R_{A B}=8 \pi G\left[T_{A B}-\frac{1}{D-2} g_{A B} T\right]
$$

where $G$ is the gravitational constant in $D$ dimensions, and $T_{A B}$ is the $D$ dimensional energy momentum tensor (capital indices run over $1,2, \ldots, \mathrm{D}$ ). by

Let us consider the $D$ dimensional spherically symmetric metric, given

$$
d s^{2}=e^{\nu(r)} d t^{2}-e^{\lambda(r)} d r^{2}-r^{2} d \Omega,
$$

where $d \Omega$ is the line element on a unit $(D-2)$ sphere.

Now, let us assume that the $D$ dimensional energy-momentum tensor has the form

$$
T_{A}^{B}=\operatorname{diag}(\rho,-p,-p, \ldots,-p),
$$

where $\rho$ is the energy density and $p$ is the pressure. With this choice the field equations (11) reduce to

$$
\begin{gathered}
e^{-\lambda(r)}\left(\frac{\lambda^{\prime}}{r}-\frac{n}{r^{2}}\right)+\frac{n}{r^{2}}=\frac{16 \pi G}{n+1} \rho, \\
e^{-\lambda(r)}\left(\frac{\nu^{\prime}}{r}+\frac{n}{r^{2}}\right)-\frac{n}{r^{2}}=\frac{16 \pi G}{n+1} p, \\
e^{-\lambda(r)}\left(\frac{\nu^{\prime \prime}}{2}+\frac{\nu^{\prime 2}}{4}-\frac{\lambda^{\prime} \nu^{\prime}}{4}-\frac{n \lambda^{\prime}+\nu^{\prime}}{2 r}-\frac{n}{r^{2}}\right)+\frac{n}{r^{2}}=0,
\end{gathered}
$$

where $n=D-3$. In the empty space surrounding the sphere of matter the solution to these equations is the exterior Schwarzschild solution in $D$ 
dimensionsf

$$
d s^{2}=\left(1-\frac{2 G M}{r^{n}}\right) d t^{2}-\frac{d r^{2}}{1-\frac{2 G M}{r^{n}}}-r^{2} d \Omega .
$$

where $M$ is interpreted as the total mass of the body. Inside the sphere this solution must be continued by a solution to (4)-(6) obtained under the conditions that the pressure be zero at the boundary of the sphere, and the metric functions be continuous across this boundary.

Equation (đ) is easily integrated into

$$
e^{-\lambda}=1-\frac{2 G m(r)}{r^{n}},
$$

where the function $m(r)$ is given by

$$
m(r)=\frac{8 \pi}{n+1} \int_{0}^{r} \rho\left(r^{\prime}\right) r^{\prime n+1} d r^{\prime}
$$

and the constant of integration has been set equal to zero to remove singularities at the origin.

The continuity of the interior metric with the exterior solution at the boundary of the sphere $r=R$, requires that $m(R)=M$. Therefore, Eq. (9) is interpreted as the gravitational mass inside a sphere of radius $r$. In what follows we will call it mass function?.

The conservation of stress energy yields

$$
p^{\prime}=-\frac{\nu^{\prime}}{2}(\rho+p) \text {. }
$$

The expression for $\nu^{\prime}$ can be obtained from equations (5) and (8)

$$
\nu^{\prime}=\frac{2 G}{n+1} \frac{m n(n+1)+8 \pi p r^{n+2}}{r\left(r^{n}-2 G m\right)} .
$$

${ }^{4}$ This line element becomes singular on $r=(2 G M)^{1 / n}$. Therefore, in $D$ dimensions, the "radius" $R$ of a spherical star, in equilibrium, must be larger than $(2 G M)^{1 / n}$. This is what gives rise to the question of how close the ratio (surface gravitational potential) $G M / R^{n}$ can approach the limiting value $1 / 2$.

${ }^{5}$ The normalization of this function is different from the mass function in $D$ dimensions as defined in Ref. [1], which is

$$
m(r)=A_{n+1} \int_{0}^{r} \rho\left(r^{\prime}\right) r^{\prime n+1} d r^{\prime}
$$

where $A_{n+1}=2 \pi^{(n+2) / 2} / \Gamma((n+2) / 2)$. 
Introducing equation (11) into equation (10) we obtain the corresponding Tolman-Oppenheimer-Volkov equation in $D$ dimensions

$$
\frac{d p}{d r}=-\frac{G(p+\rho)\left[m n(n+1)+8 \pi p r^{n+2}\right]}{(n+1) r\left(r^{n}-2 G m\right)} .
$$

This equation shows how dimensionality affects the equilibrium.

Firstly, we see that, as in $4 D$ general relativity, the pressure acts as a source of the gravitational field (because of the term proportional to $p$ added to $m$ ). Also, it is affected by the gravitational field (because $\rho$ is replaced by $(\rho+p))$. Now, equation (12) shows that both the contribution of pressure to the source and the effects of gravity on pressure are independent of the number of dimensions.

Secondly, we notice that the effects of mass do increase (decrease) with the increase (decrease) of the number of dimensions. This follows from the term $n(n+1)$, which multiplies the gravitational mass $m(r)$ in $(12)$.

Finally, for the sake of completeness, we mention that the term $1 / r^{D-2}$ in the Newtonian force is now replaced by $1 / r^{2}\left(r^{D-4}-2 G m / r\right)$.

\subsection{Perfect fluids with cosmological constant}

The behavior of perfect fluids changes qualitatively when a cosmological constant is considered. For example, in $2+1$ dimensions a black hole solution is obtained with the inclusion of a negative cosmological constant [8]. Perfect fluid stars, in $2+1$ dimensions, should collapse depending on their degree of compactification [9].

Now, we will show that in the presence of a (positive) cosmological constant the TOV equation (12) imposes a lower limit on mass and matter density.

The inclusion of the cosmological constant, $\Lambda$, in the Einstein equations is straightforward making the replacements $\rho \rightarrow \rho+\Lambda$ and $p \rightarrow p-\Lambda$. Eq. (8) becomes

$$
e^{-\lambda}=1-\frac{2 G \mu(r)}{r^{n}}
$$

where $\mu(r)$ is given by 


$$
\mu(r)=m(r)+\frac{8 \pi \Lambda r^{n+2}}{(n+1)(n+2)} .
$$

If we evaluate the $T O V$ equation on the surface of the fluid $(r=R)$ we obtain

$$
p^{\prime}(R)=\frac{-G \rho\left[n(n+1) M-16 \pi \Lambda R^{n+2} /(n+2)\right]}{(n+1) R\left(R^{n}-2 G \mu(R)\right)} .
$$

Hydrostatic equilibrium requires $p^{\prime} \leq 0$. Therefore, the right hand side of equation (15) implies a lower bound on $M$, namely

$$
M \geq \frac{16 \pi \Lambda R^{n+2}}{n(n+1)(n+2)} .
$$

This bound does not depend on the sign of $\Lambda$. It is, of course, significant when $\Lambda>0$.

The above equation implies a lower limit on the matter density. In the particular case of fluid with uniform density, $\rho=\rho_{0}$, this lower bound is given by

$$
\rho_{0} \geq \frac{2 \Lambda}{n}
$$

We note that $\rho_{0}$ decreases with the increase of the number of dimensions, which indicates that a positive cosmological constant is less effective producing repulsion in higher dimensions than in $4 D$. In other words, in $4 D$ the repulsion produced by $\Lambda>0$ is bigger and consequently more mass is needed to balance the distribution.

\section{DEGREE OF COMPACTIFICATION OF STARS IN $D$ DIMENSIONS}

\subsection{Extension of Buchdahl's Theorem to $D$ dim}

In general relativity it is well known that the equations of stellar structure for perfect fluid matter lead to the existence of an upper mass limit, viz.,

$$
\frac{G M}{R} \leq \frac{4}{9} .
$$


This result, which was first showed by Buchdahl [10], is valid under very general conditions irrespective of the equation of state. These conditions are: (i) The material of the sphere is locally isotropic. (ii) The energy density is positive and does not increase outward. The degree of compactification in spherical stars is fixed by the contribution from the Weyl curvature tensor (the "purely gravitational field energy") to the mass-energy inside the body [11, 12].

In this section we show how Buchdahl's result (18) can be extended to any number of dimensions under the same physical conditions.

First we study the isotropy condition given by Eq. (6). This condition has extensively been studied in $4 D$, where a considerable simplification is attained with the introduction of the following notation [13]

$$
e^{-\lambda}=1-\frac{2 G m(r)}{r^{n}}=Z, e^{\nu(r)}=Y^{2}, r^{2}=x
$$

and

$$
u=\int_{0}^{x} \frac{d x^{\prime}}{\sqrt{Z\left(x^{\prime}\right)}} .
$$

With this notation Eq. (6) reduces to

$$
2 \frac{d^{2} Y}{d u^{2}}=n Y G \frac{d}{d x}\left(\frac{m}{r^{n+2}}\right) .
$$

The term $m / r^{n+2}$ can be identified with the mean density of the fluid sphere in $D$ dimensions. Therefore, our second condition

$$
\frac{d \rho}{d r} \leq 0
$$

requires

$$
\frac{d}{d r}\left(\frac{m}{r^{n+2}}\right) \leq 0
$$

Now Eq. (21) gives

$$
\frac{d^{2} Y}{d u^{2}} \leq 0
$$


which means that $d Y / d u$ decreases monotonically. This in turn implies

$$
\frac{d Y}{d u} \leq \frac{Y(u)-Y(0)}{u} .
$$

Since both $Y(0)$ and $u$ are non-negative, it follows that

$$
Y^{-1} \frac{d Y}{d u} \leq \frac{1}{u}
$$

In term of the original variables this equation reads

$$
\left(1-\frac{2 G m(r)}{r^{n}}\right)^{1 / 2} \frac{d \nu}{d r} \leq r\left[\int_{0}^{r} d r^{\prime} r^{\prime}\left(1-\frac{2 G m\left(r^{\prime}\right)}{r^{\prime n}}\right)^{-1 / 2}\right]^{-1} .
$$

Using the fact that the average density decreases outward, we can evaluate the integral in (27) as follows

$$
\begin{aligned}
\int_{o}^{r} d r^{\prime} r^{\prime}\left(1-\frac{2 G m\left(r^{\prime}\right)}{r^{\prime n}}\right)^{-1 / 2} & \geq \int_{o}^{r} d r^{\prime} r^{\prime}\left(1-\frac{2 G m(r)}{r^{n+2}} r^{\prime 2}\right)^{-1 / 2} \\
& =\frac{r^{n+2}}{2 G m(r)}\left[1-\left(1-\frac{2 G m(r)}{r^{n}}\right)^{1 / 2}\right] .
\end{aligned}
$$

Now, substituting (11) and (28) into (27) we obtain

$$
\frac{n(n+1) m(r) / r^{n}+8 \pi G p r^{2}}{(n+1)\left(1-\frac{2 G m(r)}{r^{n}}\right)^{1 / 2}} \leq \frac{2 G m(r)}{r^{n}}\left[1-\left(1-\frac{2 G m(r)}{r^{n}}\right)^{1 / 2}\right]^{-1} .
$$

This equation can be simplified as follows

$$
\frac{G m(r)}{r^{D-3}} \leq \frac{1}{(D-1)^{2}}\left[(D-2)-\frac{D-1}{D-2} 8 \pi G p r^{2}+\sqrt{(D-2)^{2}+\frac{D-1}{D-2} 16 \pi G p r^{2}}\right] .
$$

Finally, the degree of compactification is obtained by evaluating this expression at the surface of the fluid $r=R$, where $p=0$. We obtain 


$$
\frac{G M}{R^{D-3}} \leq \frac{2(D-2)}{(D-1)^{2}} .
$$

This is the desired extension to $D$ dimensions of Buchdahl's theorem. Indeed for $D=4$ we recover Buchdahl's limit. It shows that $G M / R^{D-3}$ decreases with the increase of the number of dimensions. Consequently, the degree of compactification is maximum in $D=4$.

\subsection{Particular example}

The results discussed so far are model independent; they follow solely from the physical conditions (1) and (2) listed in Sec. 3.1, and the matching conditions at the boundary surface. In this section we show a simple model, with "reasonable" physical properties, that illustrates our results and indicates how (in what limit) our inequality (31) can be saturated.

We will consider the case of uniform proper density in $D$ dimensions. With $\rho=\rho_{0}$, Eqs.(9) and (20) give

$$
m(r)=\frac{8 \pi \rho_{0} r^{n+2}}{(n+1)(n+2)},
$$

and

$$
u=\frac{2}{C}[1-\sqrt{1-C x}], \quad C=\frac{16 \pi G \rho_{0}}{(n+1)(n+2)},
$$

respectively. Now, the isotropy condition (21) becomes $Y_{u u}=0$.

Therefore

$$
Y=e^{\nu / 2}=A+B[1-\sqrt{1-C x}]
$$

The constants $A, B$ and $C$ are specified by matching the interior metric to the exterior Schwarzschild solution (77). The result is

$$
\begin{gathered}
Y=e^{\nu / 2}=\left(1+\frac{n}{2}\right) \sqrt{1-\frac{2 G M}{R^{n}}}-\frac{n}{2} \sqrt{1-\frac{2 G M r^{2}}{R^{n+2}}} \\
Z=e^{-\lambda}=1-\frac{2 G M r^{2}}{R^{n+2}}
\end{gathered}
$$


For the pressure and density we find

$$
\frac{p}{\rho}=n \frac{\sqrt{1-\frac{2 G M r^{2}}{R^{n+2}}}-\sqrt{1-\frac{2 G M}{R^{n}}}}{(2+n) \sqrt{1-\frac{2 G M}{R^{n}}}-n \sqrt{1-\frac{2 G M r^{2}}{R^{n+2}}}} .
$$

Notice that $p=0$ at the boundary $r=R$ and $(p / \rho) \geq 0$. In order that the pressure may never become infinite at the origin the denominator of (37) must never vanish. This gives

$$
\frac{G M}{R^{n}}<\frac{2(1+n)}{(2+n)^{2}}=\frac{2(D-2)}{(D-1)^{2}},
$$

which is the limit (31) found from general considerations. We notice that the equality

$$
\frac{G M}{R^{n}}=\frac{2(1+n)}{(2+n)^{2}}=\frac{2(D-2)}{(D-1)^{2}},
$$

takes place in the limit when the central pressure becomes infinite. This is exactly what happens with the famous $4 / 9$ Buchdahl limit in $4-D$.

\section{CONCLUSIONS}

Equation (31) constitutes our main result. It shows how the degree of compactification depends on the number of dimensions. At the surface of the star the metric coefficient $g_{00}$ is given by $e^{\nu(R)} \geq(D-3)^{2} /(D-1)^{2}$. We see that, as a function of $D$, the minimum value of $e^{\nu(R)}$ is attained for $D=4$, viz., namely $e^{\nu(R)}=1 / 9$. For bigger number of dimensions $g_{00}(R)$ approaches unity. Since the strength of gravity depends on the deviations of the metric from its galilean values, it follows that, in more than 4 dimensions, the classical relativistic effects are weaker than in $4 D$.

This result is obviously related to the fact, elucidated by eq. (12), that the effect of mass is increased in more that 4-dim., while the effects of pressure remain the same. Consequently, in more than $4 D$ we cannot pack "too much" mass into a fixed volume without provoking gravitational collapse. In this sense $4 D$ is the optimum number of dimensions for gravity.

The questions of why does this upper limit (31) exist, and how it can be saturated, removed or at least increased are interesting questions. Previous 
investigations in $4 D$ show that the behavior of the Weyl curvature tensor plays an important role in this problem [11], [13]. Namely, that a positive (negative) contribution from the Weyl tensor tends to increase (decrease) the effective gravitational mass. Furthermore, it has been shown that Buchdahl's limit may be increased (or removed) only in the case when this contribution is negative.

Examples of reasonable physical systems showing these properties, are provided by certain types of anisotropic fluids. One would expect that similar results can be obtained in more than four dimensions.

The existence of the lower mass limit, indicated by eq. (16), in the presence of a positive cosmological constant, is not surprising. Indeed, the repulsion produced by $\Lambda>0$ should produce expansion (inflation) of an empty space. Therefore, some minimum amount of mass producing gravitational attraction and balancing the repulsion is needed for an equilibrium to exist. What is interesting here, is the role played by dimensionality. Specifically, that the minimum amount of mass depends on the number of dimensions and it is maximum in $4 D$ and decreases with the increase of dimensionality. What this suggests is that the effects of a positive cosmological constant would be more important in $4 D$ than in any other number of dimensions. This again, is a consequence of the fact that the effects of mass are increased by dimensionality.

\section{ACKNOWLEDGMENTS}

The work of N. Cruz was partially supported by Grant 195.0278 from FONDECYT (Chile) and grant from DICYT of Universidad de Santiago $\mathrm{N}^{0}$ 0497-31 CM.

\section{References}

[1] Myers, R.C. and Perry, M.T. (1986). Ann.Phys.(N.Y.) 172, 304.

[2] Dianyan, Xu. (1988). Class. Quantum Grav. 5, 871.

[3] Bañados, M; Teitelboim, C. and Zanelli, J. (1994). Phys. Rev. D49, 975.

[4] Krori, K; Borgohan, P. and Kanika, Das. (1988). Phys. Lett. A132, 321.

[5] Shen, You-Gen and Tan, Zhen-Qiang. (1988). Phys. Lett. A142, 341. 
[6] Wesson, P.S. and Ponce de Leon, J. (1992). J. Math. Phys. 33, 3883.

[7] Ponce de Leon, J. and Wesson, P.S. (1993). J. Math. Phys. 34, 4080.

[8] Bañados, M; Teitelboim, C and Zanelli, J. (1992). Phys. Rev. Lett. 69, 1849.

[9] Cruz, N. and Zanelli, J. (1995). Class. Quantum. Grav. 12, 975.

[10] Buchdahl, H.A. (1959). Phys. Rev. 116, 1027.

[11] Ponce de Leon, J. (1988). Phys. Rev.D 37, 309.

[12] Ponce de Leon, J. (1993). Gen. Rel. and Gravit. 25, 1123.

[13] Ponce de Leon, J. (1988). J. Math. Phys. 29, 197. 